\title{
Correlation of PAPP-A values with maternal characteristics, biochemical and ultrasonographic markers of pregnancy
}

\author{
Hande KAYMAKCALAN $^{1}$ (D), Ommu Gulsum UZUT $^{2}$ (D), Juho HARKONEN ${ }^{3}$ (D), Burcu BAKIR GUNGOR ${ }^{4}$ (D) \\ ${ }^{1}$ Department of Pediatrics, School of Medicine, Demiroglu Bilim University, Istanbul, Turkey. \\ ${ }^{2}$ Department of Computer Engineering, Faculty of Engineering and Architecture, Mus Alparslan University, Mus, Turkey. \\ ${ }^{3}$ Sociology, European University Institute, Florence, Italy. \\ ${ }^{4}$ Department of Computer Engineering, School of Engineering, Abdullah Gul University, Kayseri, Turkey.
}

Corresponding Author: Hande KAYMAKCALAN

E-mail: doctorhande@yahoo.com

Submitted: 09.10.2020 Accepted: 23.12.2020

\begin{abstract}
Objective: Our aim is to investigate whether there is a correlation of pregnancy-associated plasma protein A (PAPP-A) values with other variables in pregnancy and maternal characteristics.

Materials and Methods: We retrospectively analyzed the relation between the PAPP-A levels, demographics, biochemical and ultrasonographic markers of the first trimester screening of 11,842 pregnant women seen at a tertiary hospital between November 2002 and November 2008.

Results: A significant difference between PAPP-A values of the diabetic and non-diabetic pregnant women were observed ( $\mathrm{p}=0.0005$, Mann-Whitney U test). In terms of weight, crown-rump length, Beta-hCG values, significant differences were observed between low and medium level PAPP-A subgroups and between low and high level PAPP-A subgroups. PAPP-A levels were found to differ significantly between the pregnant women of Caucasian origin and other racial origins.

Conclusions: Pregnant women with different ethnic and medical backgrounds have different PAPP-A values and other markers of the aneuploidy screening. To make patient specific risk predictions, understanding these interactions and differences is important. Future studies are needed to understand the pathopyhsiology behind these differences.

Keywords: Pregnancy-associated plasma protein A (PAPP-A), Maternal characteristics, Aneuploidy screening, Biochemical markers, Ultrasonographic markers
\end{abstract}

\section{INTRODUCTION}

Pregnancy-associated plasma protein A (PAPP-A) is one of the markers used in aneuploidy screening. It can be used alone or with other markers. Used alone, low PAPP-A levels can indicate adverse pregnancy outcomes such as small for gestational age babies, preterm delivery, intrauterine growth retardation and large for gestational age babies in high PAPP-A levels [1-3]. High levels of PAPP-A (PAPP-A more than 4 multiples of median $(\mathrm{MoM})$ are rarely observed and there are a few studies on the outcomes of pregnancies with extremely high PAPP-A levels (More than 5 MoM) [4].

Previous studies demonstrated that there is an effect of different PAPP-A levels on maternal characteristics [5-8]. Westergaard et al., found statistically significant correlation between PAPP-A concentrations and maternal weight, placental weight, fetal sex and gravidity [5]. Several studies showed that PAPP-A level is higher in women of Afro-Caribbean, South Asian and East Asian racial origin, than in Caucasian women [7,8].

The relation between gestational diabetes and PAPP-A is an active research area [9]. Syngelaki et al., reported that PAPP-A levels of patients who developed gestational diabetes were 5.1-30.8\% lower than other pregnant women [10]. Lovati et al. showed that low PAPP-A was associated with gestational diabetes mellitus (DM) and lower values were found in insulin dependent diabetic women [11].

The objectives of this study with its large cohort is to identify and quantify the effects of maternal characteristics and biochemical and ultrasonographic markers of first trimester screening on PAPP-A levels to make better predictions for pregnancy outcome and therefore to give better counselling and follow up.

How to cite this article: Kaymakcalan H, Uzut O G, Harkonen J, Bakir Gungor B. Correlation of PAPP-A values with maternal characteristics, biochemical and ultrasonographic markers of pregnancy. Marmara Med J 2021:34(1):18-23. doi: 10.5472/marumj.866601 


\section{MATERIALS and METHODS}

We retrospectively collected first trimester screening data from 11,824 singleton pregnant women followed between November 2002 and November 2008 after approval by the Yale University Ethics Commitee (September 23, 2008, protocol number HIC 080.900.4229). In this study group, there were only 23 pregnancies $(0.18 \%)$ with PAPP-A above 5 MoM .

As part of first trimester aneuploidy screening, free beta human chorionic gonadotrophin (Beta-hCG) and PAPP-A measurements of pregnant women were collected. Free BetahCG and PAPP-A were measured using IMMULITE 1,000 analyzer (BioDPC, USA). The concentrations of these markers were converted to MoMs. Features such as age, weight, diabetes status, age group risk, down syndrome risk, nuchal translucency (NT), and crown-rump length (CRL) were recorded.

Mean, median, minimum and maximum values of 11,842 patients' age, weight, gestational week, crown-rump length (CRL), nuchal translucency (NT), PAPP-A, beta-hCG, age group risk and Down syndrome risk values were calculated. Log10 transformation was applied to MoM values of NT, free Beta-hCG, and PAPP-A.

\section{Statistical Aalysis}

Statistical analysis was performed on a computer using statistical program for social sciences version 22.0 (IBM SPSS Statistics Base 22.0). 11,842 patients were divided into subgroups according to PAPP-A levels, diabetes status and race. Mean, median, minimum and maximum values were recalculated. Mann-Whitney $U$ test was used to test whether the variables used in the study were significant. The significance level was set to $95 \%$. Log-transformed data were also analyzed in this study to normalize the distribution of the residuals, as required for the linear regression and as it is a widely used method in this field. P-values less than 0.05 were considered statistically significant.

\section{RESULTS}

First, we analyzed the characteristics of 11,842 patients (Table I), and the characteristics of the subgroups that we created according to the pregnant women's diabetic status (Table II). As shown in Table II, there was a significant difference between PAPP-A MoM values of the diabetic and non-diabetic pregnant women $(p=0.0005)$. For diabetic pregnant women, mean of PAPP-A MoM was 1.03, while for non-diabetic pregnant women, this value was 1.18. Same statistical difference was also observed between PAPP-A $\log 10 \mathrm{MoM}$ values of the diabetic and non-diabetic pregnant women $(\mathrm{p}=0.0005)$. For diabetic pregnant women, mean of PAPP-A log $10 \mathrm{MoM}$ was -0.06 , while for non-diabetic pregnant women, this value was 0.002 . Another statistically significant difference was found between the weights of pregnant and non-diabetic pregnant women $(\mathrm{p}<0.0001)$. For diabetic pregnant women, mean of weight was $89.4 \mathrm{~kg}$, while for non-diabetic pregnant women, this value was $70.5 \mathrm{~kg}$.

Table I. Maternal and pregnancy characteristics of study population

\begin{tabular}{lccc}
\hline \multirow{2}{*}{ Characteristics } & \multicolumn{3}{c}{ All patients } \\
\cline { 2 - 4 } Age (years) & $\mathbf{N}$ & Mean (SD) & Median (min-max) \\
\hline Weight (kg) & 11842 & $31.76 \pm 5.46$ & $32(14-53)$ \\
Gestational week & 11842 & $11.5 \pm 0.82$ & $12(10-14)$ \\
\hline CRL (mm) & 11842 & $61.3 \pm 8.5$ & $61.3(36-79.3)$ \\
NT MoM & 11842 & $1.04 \pm 0.3$ & $1(0.3-6.28)$ \\
PAPP-A MoM & 11842 & $1.1 \pm 0.7$ & $1(0.06-12.4)$ \\
Beta-hCG MoM & 11842 & $1.07 \pm 0.43$ & $1.01(0.05-5.01)$ \\
\hline $\begin{array}{l}\text { PAPP-A } \\
\text { (log10MoM) }\end{array}$ & 11842 & $0.0016 \pm 0.24$ & $0(-1.22-1.09)$ \\
Beta-hCG (log10MoM) & 11842 & $-0.00056 \pm 0.17161$ & $0.0043(-1.3-0.699)$ \\
\hline Age group risk & 11842 & $390.81 \pm 252.15$ & $340(4-1200)$ \\
Ds_risk & 11842 & $5023.38 \pm 5543.5$ & $2800(2-20000)$ \\
\hline
\end{tabular}

CRL: Fetal crown-to-rump length, NT:Nuchal translucency, hCG: human chorionic gonadotropin, MoM: Multiples of median, Ds: Down syndrome.

Table II. Maternal and pregnancy characteristics of diabetic status-based subgroups

\begin{tabular}{|c|c|c|c|c|c|c|c|}
\hline \multirow{2}{*}{ Characteristics } & \multicolumn{3}{|c|}{ Diabetic } & \multicolumn{3}{|c|}{ Non-Diabetic } & \multirow{2}{*}{$\begin{array}{l}\text { p (Mann Whitney U test) } \\
\text { Diabetic and non-diabetic }\end{array}$} \\
\hline & $\mathrm{N}$ & Mean $(S D)$ & Median (min-max) & $\mathbf{N}$ & Mean (SD) & Median (min-max) & \\
\hline Age (years) & 185 & $31.28 \pm 6.33$ & $32(16-46)$ & 11657 & $31.7 \pm 5.44$ & $32(14-53)$ & 0.385 \\
\hline Weight (kg) & 185 & $89.4 \pm 24.5$ & $85.3(47.9-182)$ & 11657 & $70.5 \pm 16.7$ & $66.6(36.5-172.4)$ & $<0.00001^{*}$ \\
\hline Gestational week & 185 & $11.5 \pm 0.85$ & $11(10-13)$ & 11657 & $11.5 \pm 0.8$ & $12(10-14)$ & 0.543 \\
\hline $\mathrm{CRL}(\mathrm{mm})$ & 185 & $60.42 \pm 9.22$ & $60.8(38-79.3)$ & 11657 & $61.3 \pm 8.5$ & $61.3(36-79.3)$ & 0.182 \\
\hline NT MoM & 185 & $1.04 \pm 0.3$ & $\begin{array}{c}1.01 \\
(0.55-2.51)\end{array}$ & 11657 & $1.04 \pm 0.3$ & $1(0.3-6.28)$ & 0.953 \\
\hline PAPP-A MoM & 185 & $1.03 \pm 0.72$ & $\begin{array}{c}0.85 \\
(0.16-5.67)\end{array}$ & 11657 & $1.18 \pm 0.7$ & $1.01(0.06-12.4)$ & $0.00049^{\star}$ \\
\hline Beta-hCG MoM & 185 & $1.07 \pm 0.43$ & $\begin{array}{c}1.01 \\
(0.16-2.86)\end{array}$ & 11657 & $1.07 \pm 0.43$ & $1.01(0.05-5.01)$ & 0.825 \\
\hline $\begin{array}{l}\text { PAPP-A } \\
(\log 10 \mathrm{MoM})\end{array}$ & 185 & $-0.06 \pm 0.26$ & $\begin{array}{c}-0.07 \\
(-0.79-0.75)\end{array}$ & 11657 & $0.002 \pm 0.24$ & $0.004(-1.22-1.09)$ & $0.00049^{*}$ \\
\hline $\begin{array}{l}\text { Beta-hCG } \\
(\log 10 \mathrm{MoM})\end{array}$ & 185 & $\begin{array}{l}-0.006 \\
\pm 0.18\end{array}$ & $\begin{array}{c}0.004 \\
(-0.79-0.45)\end{array}$ & 11657 & $\begin{array}{l}-0.0004 \\
\pm 0.17\end{array}$ & $0.0043(-1.3-0.69)$ & 0.825 \\
\hline Age group risk & 185 & $416.25 \pm 277.8$ & $360(11-890)$ & 11657 & $390.4 \pm 251.7$ & $340(4-1200)$ & 0.289 \\
\hline Ds_risk & 185 & $4431.13 \pm 5219.5$ & $240(8-20000)$ & 11657 & $5032.78 \pm 5548$ & $2800(2-20000)$ & 0.055 \\
\hline
\end{tabular}

CRL: Fetal crown-to-rump length, NT: Nuchal translucency, hCG: human chorionic gonadotropin, Ds: Down syndome, MoM: Multiples of median, ${ }^{*} U$ sing Mann-Whitney $U$ test, statistical significance at $p<0.05$. 
We then divided our dataset into the following three subgroups according to the PAPP-A levels; low PAPP-A (PAPP-A level < 4), medium PAPP-A ( $<$ PAPP-A level $<5$ ), and high PAPP-A (PAPP-A level $>5$ ). Table III identifies the characteristics of these subgroups. Significant differences were observed between low PAPP-A level subgroup and medium PAPP-A level subgroup in terms of weight, CRL, Beta - hCG MoM, Beta-HCG log10MoM values ( $p$ values of Mann-Whitney $U$ test<0.05). Similarly, significant differences were observed between low PAPP-A level subgroup and high PAPP-A level subgroup in terms of weight, Beta-hCG MoM, Beta-hCG $\log 10 \mathrm{MoM}$ values ( $\mathrm{p}$ values of Mann-Whitney U test<0.05). Mean of weight was $70.79 \mathrm{~kg}$ for low PAPP-A level subgroup, while this value was $91.84 \mathrm{~kg}$ for high PAPP-A level subgroup.

Table III. Maternal and pregnancy characteristics of PAPP-A level-based subgroup

\begin{tabular}{|c|c|c|c|c|c|c|c|c|c|c|c|c|}
\hline & \multicolumn{3}{|c|}{ Subjects with PAPP-A level $<4$ (Low) } & \multicolumn{3}{|c|}{$4<$ PAPP-A level $<5$ (Medium) } & \multicolumn{3}{|c|}{ PAPP-A level > 5 (High) } & \multicolumn{3}{|c|}{$\begin{array}{l}\text { p (Mann-Whitney U test for } \\
\text { PAPP-A levels) }\end{array}$} \\
\hline & $\mathbf{N}$ & Mean (SD) & $\begin{array}{l}\text { M e d i a n } \\
(\min -\max )\end{array}$ & $\mathrm{N}$ & Mean (SD) & $\begin{array}{l}\text { M e d i a n } \\
(\min -\max )\end{array}$ & $\mathrm{N}$ & Mean (SD) & $\begin{array}{l}\text { Median } \quad(\text { min- } \\
\max )\end{array}$ & $\begin{array}{l}\text { L o w - } \\
\text { Medium }\end{array}$ & $\begin{array}{l}\text { Low - } \\
\text { High }\end{array}$ & $\begin{array}{l}\text { Medium } \\
\text {-High }\end{array}$ \\
\hline Age (years) & 11762 & $31.76 \pm 5.46$ & $32(14-53)$ & 52 & $31.7 \pm 5.43$ & $32(21-40)$ & 28 & $32.67 \pm 4.11$ & $33.5(25-42)$ & 0.905 & 0.479 & 0.606 \\
\hline Weight (kg) & 11762 & $70.79 \pm 16.97$ & $66.6(36.5-182)$ & 52 & $77.9 \pm 19.2$ & $71.4(48.8-135)$ & 28 & $91.84 \pm 35.21$ & $90.11(47.45-161.53)$ & $0.005^{\star}$ & $0.005^{\star}$ & 0.217 \\
\hline $\begin{array}{l}\text { Gestational } \\
\text { week }\end{array}$ & 11762 & $11.55 \pm 0.82$ & $12(10-14)$ & 52 & $11.25 \pm 0.7$ & $11(10-13)$ & 28 & $11.35 \pm 0.85$ & $11(10-13)$ & 0.006 & 0.144 & 0.722 \\
\hline CRL (mm) & 11762 & $61.39 \pm 8.57$ & $61.3(36-79.3)$ & 52 & $57.07 \pm 8.53$ & $57.3(41-79.3)$ & 28 & $59.3 \pm 9.9$ & $59.1(45.2-77.3)$ & $0.001^{*}$ & 0.224 & 0.356 \\
\hline NT MoM & 11762 & $1.04 \pm 0.3$ & $1(0.3-6.28)$ & 52 & $1.1 \pm 0.34$ & $1.05(0.5-2.39)$ & 28 & $0.98 \pm 0.33$ & $0.97(0.31-1.84)$ & 0.222 & 0.327 & 0.162 \\
\hline $\begin{array}{l}\text { Beta-hCG } \\
\text { MoM }\end{array}$ & 11762 & $1.07 \pm 0.42$ & $1.01(0.07-5.01)$ & 52 & $1.34 \pm 0.5$ & $1.21(0.37-3.02)$ & 28 & $1.46 \pm 0.73$ & $1.37(0.05-3.41)$ & $<0.0001^{\star}$ & $0.001^{*}$ & 0.661 \\
\hline $\begin{array}{l}\text { Beta-hCG } \\
(\log 10 \mathrm{MoM})\end{array}$ & 11762 & $-0.001 \pm 0.17$ & $\begin{array}{lrrrr}0 & 0 & 0 & 0 & 4 \\
(-1.15-0.69)\end{array}$ & 52 & $0.09 \pm 0.17$ & $\begin{array}{l}0.08(-0.43- \\
0.48)\end{array}$ & 28 & $0.08 \pm 0.32$ & $0.13(-1.3-0.5)$ & $<0.0001^{*}$ & $0.001^{*}$ & 0.661 \\
\hline Age group risk & 11762 & $390.9 \pm 252.1$ & $340(4-1200)$ & 52 & $393.9 \pm 261.9$ & $340(50-850)$ & 28 & $341.6 \pm 210.3$ & $265(35-760)$ & 0.995 & 0.404 & 0.505 \\
\hline Ds_risk & 11762 & $5011.6 \pm 5532.4$ & $2800(2-20000)$ & 52 & $6983 \pm 7096.1$ & $3050(20-20000)$ & 28 & $6321.78 \pm 6105.6$ & $3100(310-20000)$ & 0.081 & 0.146 & 0.972 \\
\hline
\end{tabular}

CRL: Fetal crown-to-rump length, NT: Nuchal translucency, hCG: human chorionic gonadotropin, Ds: Down syndome, MoM: Multiples of median, ${ }^{*} U s i n g ~ M a n n-W h i t n e y$ $U$ test, statistical significance at $p<0.05$.

Table IV. Maternal and pregnancy characteristics of racial origin based subgroups

\begin{tabular}{|c|c|c|c|c|c|c|c|c|c|c|c|c|}
\hline & & Caucasian Pati & ents $(C)$ & & ro-Caribbean P & atients (A) & Pati & ents of Other Et & hnicities (0) & $\begin{array}{r}P(\text { Mann } \\
\text { diabe }\end{array}$ & $\begin{array}{l}\text { Whitney } \mathrm{U} \\
\text { tic yes and }\end{array}$ & $\begin{array}{l}\text { test for } \\
\text { no) }\end{array}$ \\
\hline & $\mathbf{N}$ & Mean (SD) & $\begin{array}{l}\text { Median } \\
(\text { min-max })\end{array}$ & $\mathrm{N}$ & Mean (SD) & $\begin{array}{c}\text { Median } \\
(\text { min-max })\end{array}$ & $\mathbf{N}$ & Mean (SD) & $\begin{array}{c}\text { Median } \\
(\text { min-max })\end{array}$ & C-A & $\mathrm{C}-\mathrm{O}$ & A-O \\
\hline Age (years) & 8807 & $32.3 \pm 5.11$ & $33(15-53)$ & 1046 & $29.4 \pm 6.37$ & $29(15-46)$ & 1989 & $30.4 \pm 5.82$ & $31(14-45)$ & $<0.0001^{*}$ & $<0.0001^{*}$ & $<0.0001^{*}$ \\
\hline Weight (kg) & 8807 & $70.7 \pm 16.3$ & $\begin{array}{c}66.6(40.15- \\
172.4)\end{array}$ & 1046 & $81.5 \pm 20.9$ & $\begin{array}{c}78.02 \\
(45.17-182.06)\end{array}$ & 1989 & $65.7 \pm 15.6$ & $\begin{array}{c}62.05(36.5- \\
161.5)\end{array}$ & $<0.0001^{\star}$ & $<0.0001^{*}$ & $<0.0001^{*}$ \\
\hline $\begin{array}{l}\text { Gestational } \\
\text { week }\end{array}$ & 8807 & $11.5 \pm 0.8$ & $11(10-14)$ & 1046 & $11.7 \pm 0.86$ & $12(10-13)$ & 1989 & $11.6 \pm 0.8$ & $12(10-14)$ & $<0.0001^{*}$ & $<0.0001^{*}$ & $0.002^{*}$ \\
\hline CRL (mm) & 8807 & $61.2 \pm 8.49$ & $61.1(36-79.3)$ & 1046 & $62.16 \pm 8.87$ & $62.1(36-79.3)$ & 1989 & $61.5 \pm 8.7$ & $61.5(36.1-79.3)$ & $0.001^{*}$ & 0.089 & 0.095 \\
\hline NT MoM & 8807 & $1.04 \pm 0.32$ & $1(0.3-6.28)$ & 1046 & $0.98 \pm 0.25$ & $\begin{array}{c}0.95(0.31- \\
2.78)\end{array}$ & 1989 & $1.06 \pm 0.32$ & $1.02(0.41-4.74)$ & $<0.0001^{\star}$ & 0.04 & $<0.0001^{\star}$ \\
\hline $\begin{array}{l}\text { PAPP-A } \\
\text { MoM }\end{array}$ & 8807 & $1.14 \pm 0.66$ & $\begin{array}{c}0.98(0.06- \\
8.32)\end{array}$ & 1046 & $1.45 \pm 1.01$ & $\begin{array}{c}1.21(0.16- \\
12.4)\end{array}$ & 1989 & $1.18 \pm 0.73$ & $1(0.08-6.24)$ & $<0.000001^{\star}$ & 0.269 & $<0.0001^{\star}$ \\
\hline $\begin{array}{l}\text { Beta-hCG } \\
\text { MoM }\end{array}$ & 8807 & $1.06 \pm 0.41$ & $1(0.09-4.37)$ & 1046 & $1.21 \pm 0.52$ & $\begin{array}{c}1.11(0.05- \\
5.01)\end{array}$ & 1989 & $1.07 \pm 0.42$ & $1.01(0.11-3.3)$ & $<0.0001^{\star}$ & 0.170 & $<0.0001^{\star}$ \\
\hline $\begin{array}{l}\text { PAPP-A } \\
(\log 10 \mathrm{MoM})\end{array}$ & 8807 & $-0.006 \pm 0.23$ & $\begin{array}{c}-0.0087(-1.22- \\
0.92)\end{array}$ & 1046 & $0.075 \pm 0.27$ & $\begin{array}{c}0.08(-0.79- \\
1.09)\end{array}$ & 1989 & $0.00038 \pm 0.25$ & $0(-1.09-0.79)$ & $<0.0001^{\star}$ & 0.269 & $<0.0001^{\star}$ \\
\hline $\begin{array}{l}\text { Beta - hCG } \\
(\log 10 \mathrm{MoM})\end{array}$ & 8807 & $-0.006 \pm 0.16$ & $0(-1.04-0.64)$ & 1046 & $0.04 \pm 0.18$ & $0.04(-1.3-0.69)$ & 1989 & $0.00043 \pm 0.16$ & $\begin{array}{c}0.004(-0.95- \\
0.51)\end{array}$ & $<0.0001^{*}$ & 0.170 & $<0.0001^{\star}$ \\
\hline $\begin{array}{l}\text { Age group } \\
\text { risk }\end{array}$ & 8807 & $365.09 \pm 240.05$ & $310(4-900)$ & 1046 & $494.17 \pm 282.6$ & $525(11-900)$ & 1989 & $450.36 \pm 263.25$ & $430(14-1200)$ & $<0.0001^{\star}$ & $<0.0001^{*}$ & $<0.0001^{\star}$ \\
\hline Ds_risk & 8807 & $4679.9 \pm 5271.3$ & $2600(2-20000)$ & 1046 & $6660.5 \pm 6405.7$ & $\begin{array}{c}4300(11- \\
20000)\end{array}$ & 1989 & $5683.2 \pm 5998.2$ & $3200(2-20000)$ & $<0.0001^{*}$ & $<0.0001^{*}$ & $<0.0001^{\star}$ \\
\hline
\end{tabular}

CRL: Fetal crown-to-rump length, NT: Nuchal translucency, hCG: human chorionic gonadotropiN, Ds: Down syndome, MoM: Multiples of median, ${ }^{*}$ Using Mann-Whitney $U$ test, statistical significance at $p<0.05$. 
We also divided our dataset into the following three subgroups according to the racial origin. $74.3 \%, 8.8 \%, 16.8 \%$ of the 11,842 subjects were Caucasian, Afro-Caribbean, other, respectively. Table IV summarizes the characteristics of these subgroups. Significant differences were observed between all pairs in terms of weight, gestational week, NT MoM, PAPP-A MoM, Beta-hCG MoM, PAPP-A log10 MoM, Beta-hCG MoM, age group risk, Down syndrome risk values. There was a significant difference in CRL between the Caucasian and Afro-Caribbean subgroups
( $p<0.05$ ). While the mean of CRL was 61.1 for Caucasian subgroup, this value was 62.16 for Afro-Caribbean subgroup.

We looked at the correlation between fetus NT values and PAPP-A MoM values of pregnant women whose PAPP-A MoM values were grouped as low-normal (less than 4), high (between 4 and 5) and very high (greater than 5) (Figure 1). There was no significant correlation between PAPP-A MoM and fetus NT measurement in these groups ( $r=0.04,0.4,0.1$ for PAPP-A lownormal, high, very high subgroups, respectively).

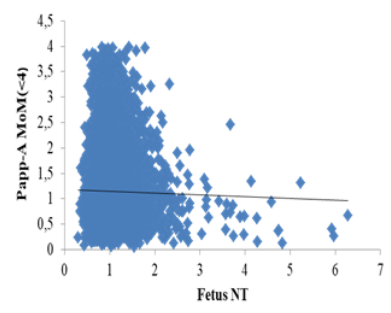

(a)

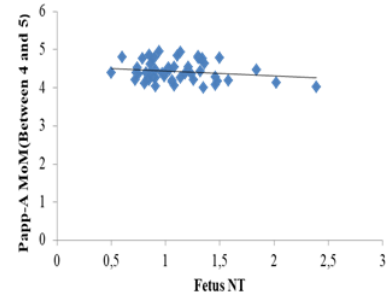

(b)

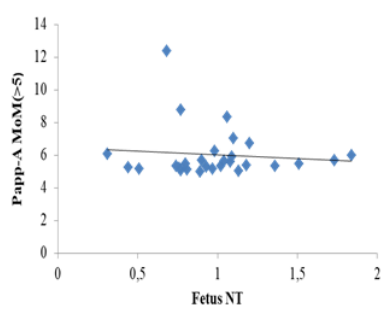

(c)

Figure 1. Correlation between fetus nuchal translucency (NT) values and PAPP-A MoM (multiples of median) values of pregnant women. PAPP-A MoM values were grouped as (a) low-normal (less than 4), (b) high (between 4 and 5) and (c) very high (greater than 5).

In addition no correlation was observed between PAPP-A MoM and NT values for racial origin groups $(r=0.009,0.029,0.029$, for Caucasians, Afro-Caribbean's, other ethnicities, respectively) (Figure
2). There was no correlation between PAPP-A MoM and CRL values for racial origin groups ( $\mathrm{r}=0.04,0.06,0.03$ for Caucasians, Afro-Caribbeans, other ethnicities, respectively) (Figure 3).

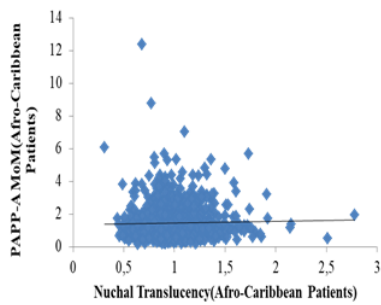

(a)

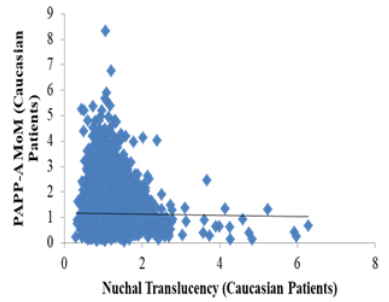

(b)

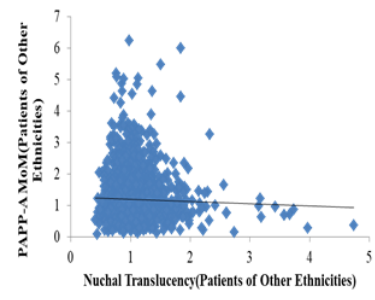

(c)

Figure 2. Correlation between PAPP-A MoM (multiples of median) values and nuchal translucency (NT) values for different racial origin groups, i.e. (a) Afro-Caribbean Patients, b) Caucasian Patients, c) Other Patients

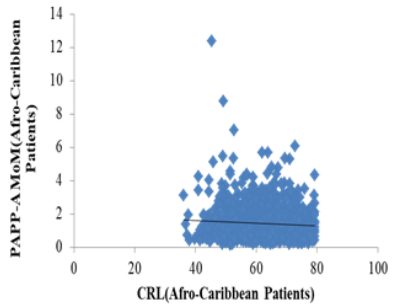

(a)

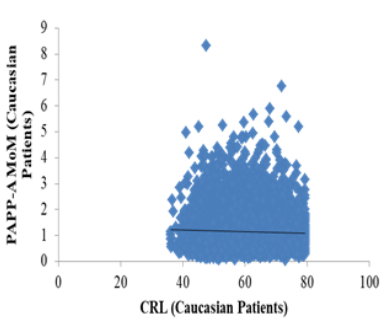

(b)

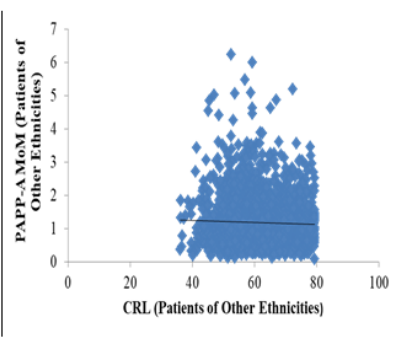

(c)

Figure 3. Correlation between PAPP-A MoM (multiples of median) values and fetal crown-to-rump length (CRL) values for different racial origin groups, i.e., (a) Afro-Caribbean Patients, b) Caucasian Patients, c) Other Patients 


\section{DISCUSSION}

Pregnancy-associated plasma protein A values are used in predicting pregnancy outcomes, so using accurate cut-off values according to patient's characteristics is important to avoid misinterpretation of results. We found similar results with the previous studies [5-7], that there was a racial difference; Caucasians had lower PAPP-A values than Afro-Caribbeans and other racial groups. In aneuploidy screening one does not fit all, and more studies are needed to make specific charts for different ethnic groups.

Gestational diabetes is another factor that affects PAPP-A levels. Again in line with previous studies [8,9], we also found that PAPP-A levels were significantly lower in pregnant women with diabetes. As expected, the weights of the women in the diabetes group were higher than the non-diabetics regardless of ethnic background. Pregnant women must be counseled about healthy diet.

Pregnancy-associated plasma protein A is a protease for insulin like growth factors (IGF) and acts as an important regulator in their function [10]. IGFs stimulate growth and decrease glucose levels. It is therefore reasonable to observe low PAPP-A levels in diabetic pregnant women.

When we looked at correlations between CRL, NT and ethnic differences, we found that there was a small but significant difference in NT and CRL measurements between fetuses of different ethnic origin. Intrauterine fetal growth charts developed by Eunice Kennedy Shriver National Institute of Child Health and Human Development (NICHD) [12], INTERGROWTH [13] and WHO [14] also showed that the percentile cut-points for SGA and LGA varied among the studies. Both environmental and genetic factors are hypothesized to cause differences in body size and proportion [15], but still determinants of fetal growth are not fully understood [16,17].

Our study with its large cohort of pregnant women coming from different ethnic origins provides useful data for examining the relationship between certain demographic factors, diabetes, CRL, NT and PAPP-A levels. Adjusting the normal values for different backgrounds is important to make accurate patient specific risk predictions [18]. Understanding its interaction with other pregnancy markers and demographics may help us better understand PAPP-A physiology and function in pregnancy.

\section{Acknowledgments}

We would like to thank Prof. James McGrath and Prof. Maurice J. Mahoney at Yale University Department of Medical Genetics for their valuable guidance and input.

\section{Compliance with Ethical Standards}

Ethical Approval: The study was approved by Yale University, School of Medicine Ethics Committee (September 23, 2008, protocol number HIC 080.900.4229).

Financial Support: The authors have no relevant financial information to disclose.
Conflict of Interest: The authors have no potential conflicts to disclose.

Author contributions:Research idea, data collection, literature search, reviewing of analysis results, writing: H.K.,

Data mining, statistical analysis, drawing the tables and figures, writing, literature search: O.G.U.,

Statistical analysis, literature search: J.H.,

Data mining, statistical analysis, reviewing and editing of the manuscript, literature search: B.B.G. We confirm that the final version of the article has been read and approved by all named authors.

\section{REFERENCES}

[1] Smith GC, Stenhouse EJ, Crossley JA, Aitken DA, Cameron $\mathrm{AD}$, Connor JM. Early pregnancy levels of pregnancyassociated plasma protein a and the risk of intrauterine growth restriction, premature birth, preeclampsia, and stillbirth. J Clin Endocrinol Metab 2002;87:1762-7. doi: 10.1210/ jcem.87.4.8430.

[2] Yaron Y, Heifetz S, Ochshorn Y, Lehavi O, Orr-Urtreger A. Decreased first trimester PAPP-A is a predictor of adverse pregnancy outcome. Prenat Diagn 2002;22:778-82. doi: 10.1002/pd.407.

[3] Hanita O, Roslina O, Azlin MI. Maternal level of pregnancyassociated plasma protein $\mathrm{A}$ as a predictor of pregnancy failure in threatened abortion. Malays J Pathol 2012;34:145-51.

[4] Cuckle H, Arbuzova S, Spencer K, et al. Frequency and clinical consequences of extremely high maternal serum PAPP-A levels. Prenat Diagn 2003;23:385-8. doi: 10.1002/pd.600.

[5] Westergaard JG, Teisner B, Grudzinskas JG. Serum PAPP-A in normal pregnancy: relationship to fetal and maternal characteristics. Arch Gynecol 1983;233:211-5. doi: 10.1007/ BF02114602.

[6] Wright D, Silva M, Papadopoulos S, Wright A, Nicolaides KH. Serum pregnancy-associated plasma protein-A in the three trimesters of pregnancy: effects of maternal characteristics and medical history. Ultrasound Obstet Gynecol 2015;46:4250. doi: 10.1002/uog.14870.

[7] Browne JL, Klipstein-Grobusch K, Koster MP, et al. Pregnancy associated plasma protein-a and placental growth factor in a Sub-Saharan African population: A nested cross-sectional study. PLoS ONE 2016;11:e0159592. doi: 10.1371/journal. pone.0159592.

[8] Donovan BM, Nidey NL, Jasper EA, et al. First trimester prenatal screening biomarkers and gestational diabetes mellitus: A systematic review and meta-analysis. PLoS ONE 2018;13: e0201319. doi.org/10.1371/journal.pone.0201319

[9] Syngelaki A, Kotecha R, Pastides A, Wright A, Nicolaides $\mathrm{KH}$. First-trimester biochemical markers of placentation in screening for gestational diabetes mellitus. Metabolism 2015;64:1485-9. doi: 10.1016/j.metabol.2015.07.015.

[10] DiPrisco B, Kumar A, Kalra B, et al. Placental proteases PAPP-A and PAPP-A2, the binding proteins they cleave (IGFBP-4 and 
- 5), and IGF-I and IGF-II: Levels in umbilical cord blood and associations with birth weight and length. Metabolism 2019;100:153959. doi: 10.1016/j.metabol.2019.153959.

[11] Lovati E, Beneventi F, Simonetta M, et al. Gestational diabetes mellitus: Including serum pregnancy-associated plasma protein-A testing in the clinical management of primiparous women? A case-control study. Diabetes Res Clin Pract 2013;100:340-7. doi: 10.1016/j.diabres.2013.04.002

[12] Grewal J, Grantz KL, Zhang C, et al. Cohort profile: NICHD fetal growth studies-singletons and twins. Int J Epidemiol 2018;47:25-25l. doi:10.1093/ije/dyx161

[13] Villar J, Papageorghiou AT, Pang R, et al. The likeness of fetal growth and newborn size across non-isolated populations in the INTERGROWTH-21st Project: the Fetal Growth Longitudinal Study and Newborn Cross-Sectional Study. Lancet Diabetes Endocrinol 2014;2:781-92. doi:10.1016/ S2213-8587(14)70121-4

[14] Kiserud T, Piaggio G, Carroli G, et al. The World Health Organization Fetal Growth Charts: A Multinational Longitudinal Study of Ultrasound Biometric Measurements and Estimated Fetal Weight [published correction appears in PLoS Med 2017;14 (3):e1002284] [published correction appears in PLoS Med 2017;14 (4):e1002301]. PLoS Med 2017;14(1):e1002220. Published 2017 Jan 24. doi:10.1371/ journal.pmed.1002220

[15] Bogin B, Varela-Silva MI. Leg length, body proportion, and health: a review with a note on beauty. Int J Environ Res Public Health 2010;7:1047-75. doi:10.3390/ijerph7031047

[16] Clausson B, Lichtenstein P, Cnattingius S. Genetic influence on birthweight and gestational length determined by studies in offspring of twins. BJOG 2000;107:375-81. doi:10.1111/j.1471-0528.2000.tb13234.x.

[17] Lunde A, Melve KK, Gjessing HK, Skjaerven R, Irgens LM. Genetic and environmental influences on birth weight, birth length, head circumference, and gestational age by use of population-based parent-offspring data. Am J Epidemiol 2007;165:734-41. doi:10.1093/aje/kwk107

[18] Grantz KL, Hediger ML, Liu D, Buck Louis GM. Fetal growth standards: the NICHD fetal growth study approach in context with INTERGROWTH-21st and the World Health Organization Multicentre Growth Reference Study. Am J Obstet Gynecol 2018;218(2S):S641-S655.e28. doi:10.1016/j. ajog.2017.11.593 